\title{
Deciphering Exceptional Preservation of Fossils Using Trace Elemental Imaging
}

\author{
P Gueriau $^{1,2}$ and L Bertrand $d^{2,3}$ \\ 1. CR2P, UMR 7207 CNRS, MNHN, UPMC, Paris, France. \\ 2. IPANEMA, USR 3461 CNRS, MCC, Gif-sur-Yvette, France. \\ 3. SOLEIL synchrotron, Gif-sur-Yvette, France.
}

Recently synchrotron-based X-ray fluorescence (XRF) is used to map elemental distributions (at minor-to-trace levels) on entire fossils, based on the collection of integrated intensity in preselected spectral regions of interest. These studies particularly focused on organics and metal distributions such as copper and zinc in fossil bird feathers and lizard skins [1-3]. Using a similar but different approach, we imaged a wide range of well-preserved fossils from the middle Devonian ( $-390 \mathrm{Myr})$ to the late Cretaceous (-100 Myr), including plants, invertebrates and vertebrates. Our approach is based on the collection of full-range XRF spectra coupled to spectral decomposition for all constitutive elements or a much faster divergence based statistical analysis. We deeply investigated on trace elements, particularly strontium, yttrium and rare earth elements (REEs), known to be present in significant quantities in fossil bones, teeth and sedimentary apatites [4], and used in paleontological, paleoenvironmental and taphonomic research as their distribution and fractionation depend on fossilization and diagenesis processes [4-5]. The contrasting elemental distributions greatly improved the discrimination of hard tissues (bones, carapaces or cuticles) from both the sedimentary matrix and the fossilized soft tissues on the basis of variations in elemental relative concentrations (figure 1) [6].

Although the compression of their anatomical features during fossilization makes harder the interpretation of well-preserved flattened fossils from sedimentary matrix, it is of paramount interest for taxonomic, phylogenetic, taphonomic and environmental studies of exceptional preservation deposits that are unique witnesses of the ancient traces of life on Earth. Discriminating between skeletal and soft tissues therefore appears very promising as it allows visualizing anatomical features that are hidden under well-preserved decay prone tissues or an unpreparable sedimentary matrix (figure 1, see particularly the second antennae and the legs in the fossil shrimp, and the entire skull together with vertebrae in the fossil fish) [6].

Furthermore, insights on trace elements amounts open new ways for paleoenvironmental and taphonomical studies, as they simultaneously reflect the connectivity of the environmental water network, local redox, the specific surface area of the bioapatite nanocrystals and physico-chemical conditions and properties of substituted apatite [4, 7-9]. Contrasted elemental signatures therefore evidence differences in initial compositions, concentrations, sorption and/or substitution rates, providing relevant information on fossilization processes. Ongoing work is now focused on the preferential incorporation of the contrasting elements through X-ray absorption spectroscopy.

\section{References:}

[1] U Bergmann et al, PNAS 107 (2010), 9060-9065.

[2] R Wogelius et al, Science 333 (2011), 1622-1626.

[3] N Edwards et al, Proceedings of the Royal Society B: Biological Sciences 278 (2011), 3209-3218.

[4] D Herwartz et al, Geochimica et Cosmochimica Acta 103 (2013), 161-183. 
[5] CN Trueman and MJ Benton, Geology 25 (1997), 263-266.

[6] P Gueriau et al, PLoS One 9 (2014), e86946.

[7] L Kocsis et al, Geochimica et Cosmochimica Acta 74 (2010), 6077-6092.

[8] CN Trueman et al, Palaeogeography, Palaeoclimatology, Palaeoecology 310 (2011), 124-132.

[9] CN Trueman, Palaeontology 56 (2013), 475-486.

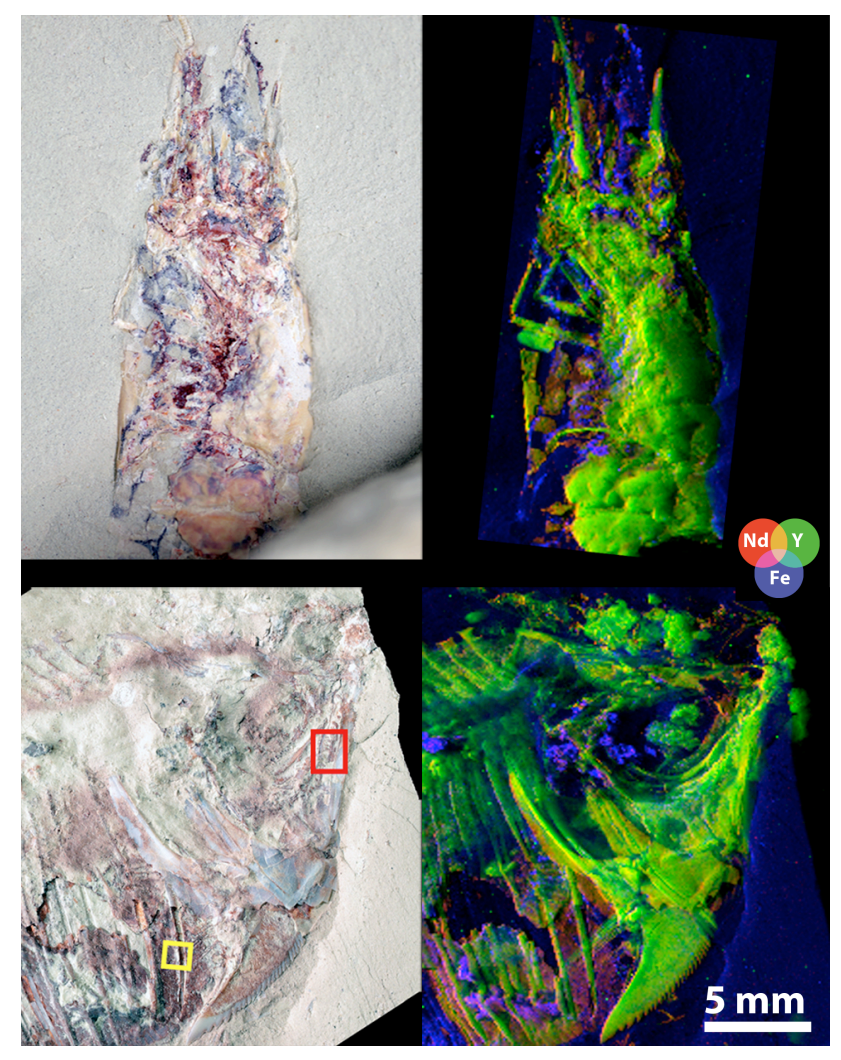

Figure 1. Synchrotron X-ray fluorescence mapping of major-to-trace elements in fossil shrimp (top) and fish (bottom) from the OT1 Lagerstätte (Upper Cretaceous, -100 Myr, Morocco). Optical photographs and false color overlays of iron (blue) and two rare earth elements (neodymium, red and yttrium, green) distributions reconstructed from a full spectral decomposition of the data. 